九州大学学術情報リポジトリ

Kyushu University Institutional Repository

\title{
Deductive Inference for the Interiors and Exteriors of Horn Theories
}

Makino, Kazuhisa

Department of Mathematical Informatics, University of Tokyo

Ono, Hirotaka

Department of Computer Science and Communication Engineering, Kyushu University

http://hdl. handle. net/2324/14863

出版情報: Lecture Notes in Computer Science. 5369，pp.390-401，2008-12-11. Springer バージョン：

権利関係 : 


\title{
Deductive Inference for the Interiors and Exteriors of Horn Theories ${ }^{\star}$
}

\author{
Kazuhisa Makino ${ }^{1}$ and Hirotaka Ono ${ }^{2}$ \\ 1 Department of Mathematical Informatics, University of Tokyo, Tokyo, 113-8656, Japan. \\ makino@mist.i.u-tokyo.ac.jp \\ 2 Department of Computer Science and Communication Engineering, Kyushu University, \\ Fukuoka, 819-0395, Japan. ono@csce.kyushu-u.ac.jp
}

\begin{abstract}
In this paper, we investigate the deductive inference for the interiors and exteriors of Horn knowledge bases, where the interiors and exteriors were introduced by Makino and Ibaraki [11] to study stability properties of knowledge bases. We present a linear time algorithm for the deduction for the interiors and show that it is co-NP-complete for the deduction for the exteriors. Under modelbased representation, we show that the deduction problem for interiors is NPcomplete while the one for exteriors is co-NP-complete. As for Horn envelopes of the exteriors, we show that it is linearly solvable under model-based representation, while it is co-NP-complete under formula-based representation. We also discuss the polynomially solvable cases for all the intractable problems.
\end{abstract}

\section{Introduction}

Knowledge-based systems are commonly used to store the sentences as our knowledge for the purpose of having automated reasoning such as deduction for them (see e.g., [1]). Deductive inference is a fundamental mode of reasoning, and usually abstracted as follows: Given the knowledge base $K B$, assumed to capture our knowledge about the domain in question, and a query $\chi$ that is assumed to capture the situation at hand, decide whether $K B$ implies $\chi$, denoted by $K B \vDash \chi$, which can be understood as the question: "Is $\chi$ consistent with the current state of knowledge?"

In this paper, we consider the interiors and exteriors of knowledge base. Formally, for a given positive integer $\alpha$, the $\alpha$-interior of $K B$, denoted by $\sigma_{-\alpha}(K B)$, is a knowledge that consists of the models (or assignments) $v$ satisfying that the $\alpha$-neighbors of $v$ are all models of $K B$, and the $\alpha$-exterior of $K B$, denoted by $\sigma_{\alpha}(K B)$, is a knowledge that consists of the models $v$ satisfying that at least one of the $\alpha$-neighbors of $v$ is a model of $K B$ [11]. Intuitively, the interior consists of the models $v$ that strongly satisfy $K B$, since all neighbors of $v$ are models of $K B$,

\footnotetext{
* This work is supported in part by the Grant-in-Aid of the Ministry of Education, Science, Sports and Culture of Japan and by the Asahi glass foundation.
} 
while the exterior consists of the models $v$ that weakly satisfy $K B$, since at least one of the $\alpha$-neighbors of $v$ is a model of $K B$. Here we note that $v$ might not satisfy $K B$, even if we say that it weakly satisfies $K B$. As mentioned in [11], the interiors and exteriors of knowledge base merit study in their own right, since they shed light on the structure of knowledge base. Moreover, let us consider the situation in which knowledge base $K B$ is not perfect in the sense that some sentences in $K B$ are wrong and/or some are missing in $K B$ (see also [11]).

Suppose that we use $K B$ as a knowledge base for automated reasoning, say, duductive inference $K B \vDash \chi$. Since $K B$ does not represent real knowledge $K B^{*}$, the reasoning result is no longer true. However, if we use the interior $\sigma_{-\alpha}(K B)$ of $K B$ as a knowledge base and have $\sigma_{-\alpha}(K B) \not \neq \chi$, then we can expect that the result is ture for real knowledge $K B^{*}$, since $\sigma_{-\alpha}(K B)$ consists of models which strongly satisfy $K B$. On the other hand, if we use the exterior $\sigma_{\alpha}(K B)$ of $\mathrm{KB}$ as a knowledge base and have $\sigma_{\alpha}(K B) \vDash \chi$, then we can expect that the result is ture for real knowledge $K B^{*}$, since $\sigma_{\alpha}(K B)$ consists of models which weakly satisfy $K B$. In this sense, the interiors and exteriors help to have safe reasoning.

Main problems considered. In this paper, we study the deductive inference for the interiors and exteriors of propositional Horn theories, where Horn theories are ubiquitous in Computer Science, cf. [14], and are of particular relevance in Artificial Intelligence and Databases. It is known that important reasoning problems like deductive inference and satisfiability checking, which are intractable in general, are solvable in linear time for Horn theories (cf. [3]).

More precisely, we address the following problems:

- Given a Horn theory $\Sigma$, a clause $c$, and an integer $\alpha>0$, we consider the problems of deciding if deductive queries hold for the $\alpha$-interior and exterior of $\Sigma$, i.e., $\sigma_{-\alpha}(\Sigma) \vDash c$ and $\sigma_{\alpha}(\Sigma) \vDash c$. It is well-known [3] that a deductive query for a Horn theory can be answered in linear time. Note that it is intractable to construct the interior and exterior for a Horn theory [11,13], and hence a direct method (i.e., first construct the interior (or exterior) and then check a deductive query) is not possible efficiently.

- We contrast traditional formula-based (syntactic) with model-based (semantic) representation of Horn theories. The latter form of representation has been proposed as an alternative form of representing and accessing a logical knowledge base, cf. [2, 4, 5, 7, 8, 6, 9, 10]. In model-based reasoning, $\Sigma$ is represented by a subset of its models $\mathcal{M}$, which are commonly called characteristic models. As shown in [7], the deductive inference can be solved in polynomial time, given its characteristic models.

- Finally, we consider Horn approximations for the exteriors of Horn theories. Note that the interiors of Horn theories are Horn, while the exteriors might not 
be Horn. We deal with the least upper bounds, called the Horn envelopes [16], for the exteriors of Horn theories.

Main results. We investigate the problems mentioned above from an algorithmical viewpoint. For all the problems, we provide either polynomial time algorithms or proofs of the intractability; thus, our work gives a complete picture of the tractability/intractability frontier of deduction for interiors and exteriors of Horn theories. Our main results can be summarized as follows (see Figure 1).

- We present a linear time algorithm for the deduction for the interiors of a given Horn theory, and show that it is co-NP-complete for the deduction for the exteriors. Thus, the positive result for ordinary deduction for Horn theories extends to the interiors, but does not to the exteriors. We also show that the deduction for the exteriors is possible in polynomial time, if $\alpha$ is bounded by a constant or if $|N(c)|$ is bounded by a logarithm of the input size, where $N(c)$ corresponds to the set of negative literals in $c$.

- Under model-based representation, we show that the consistency problem and the deduction for the interiors of Horn theories are both co-NP-complete. As for the exteriors, we show that the deduction is co-NP-complete. We also show that the deduction for the interiors is possible in polynomial time if $\alpha$ is bounded by a constant, and so is for the exteriors, if $\alpha$ or $|P(c)|$ is bounded by a constant, or if $|N(c)|$ is bounded by a logarithm of the input size, where $P(c)$ corresponds to the set of positive literals in $c$.

- As for Horn envelopes of the exteriors of Horn theories, we show that it is linearly solvable under model-based representation, while it is co-NP-complete under formula-based representation. The former contrasts to the negative result for the exteriors. We also present a polynomial algorithm for formula-based representation, if $\alpha$ is bounded by a constant or if $|N(c)|$ is bounded by a logarithm of the input size.

\begin{tabular}{l|ccc}
\hline & Interiors & Exteriors & Envelopes of Exteriors \\
\hline Formula-Based & $\mathrm{P}$ & co-NP-complete $^{\star}$ & co-NP-complete $^{\star}$ \\
\hline Model-Based & NP-complete $^{\dagger}$ & co-NP-complete $^{\ddagger}$ & $\mathrm{P}$ \\
\hline
\end{tabular}

*: It is polynomially solvable, if $\alpha=O(1)$ or $|N(c)|=O(\log \|\Sigma\|)$.

: It is polynomially solvable, if $\alpha=O(1)$.

$\ddagger$ It is polynomially solvable, if $\alpha=O(1),|P(c)|=O(1)$, or $|N(c)|=O(\log n|\operatorname{chr}(\Sigma)|)$.

Fig. 1. Complexity of the deduction for interiors and exteriors of Horn theories 
The rest of the paper is organized as follows. In the next section, we review the basic concepts and fix notations. Sections 3 and 4 investigate the deductive inference for the interiors and exteriors of Horn theories. Section 5 considers the deductive inference for the envelopes of the exteriors of Horn theories. Most of the proofs are omitted due to space limitation. Interested readers can find the omitted parts in [12], which is a technical report version of the paper.

\section{Preliminaries}

Horn Theories. We assume a standard propositional language with atoms $A t=$ $\left\{x_{1}, x_{2}, \ldots, x_{n}\right\}$, where each $x_{i}$ takes either value 1 (true) or 0 (false). A literal is either an atom $x_{i}$ or its negation, which we denote by $\bar{x}_{i}$. The opposite of a literal $\ell$ is denoted by $\bar{\ell}$, and the opposite of a set of literals $L$ by $\bar{L}=\{\bar{\ell} \mid \ell \in L\}$. Furthermore, Lit $=A t \cup \overline{A t}$ denotes the set of all literals.

A clause is a disjunction $c=\bigvee_{i \in P(c)} x_{i} \vee \bigvee_{i \in N(c)} \bar{x}_{i}$ of literals, where $P(c)$ and $N(c)$ are the sets of indices whose corresponding variables occur positively and negatively in $c$ and $P(c) \cap N(c)=\emptyset$. Dually, a term is conjunction $t=$ $\bigwedge_{i \in P(t)} x_{i} \wedge \bigwedge_{i \in N(t)} \bar{x}_{i}$ of literals, where $P(t)$ and $N(t)$ are similarly defined. We also view clauses and terms as sets of literals. A conjunctive normal form $(C N F)$ is a conjunction of clauses. A clause $c$ is Horn, if $|P(c)| \leq 1$. A theory $\Sigma$ is any set of formulas; it is Horn, if it is a set of Horn clauses. As usual, we identify $\Sigma$ with $\varphi=\bigwedge_{c \in \Sigma} c$, and write $c \in \varphi$ etc. It is known [3] that the deductive query for a Horn theory, i.e., deciding if $\Sigma \models c$ for a clause $c$ is possible in linear time.

We recall that Horn theories have a well-known semantic characterization. A model is a vector $v \in\{0,1\}^{n}$, whose $i$-th component is denoted by $v_{i}$. For a model $v$, let $O N(v)=\left\{i \mid v_{i}=1\right\}$ and $O F F(v)=\left\{i \mid v_{i}=0\right\}$. The value of a formula $\varphi$ on a model $v$, denoted $\varphi(v)$, is inductively defined as usual; satisfaction of $\varphi$ in $v$, i.e., $\varphi(v)=1$, will be denoted by $v \vDash \varphi$. The set of models of a formula $\varphi$ (resp., theory $\Sigma$ ), denoted by $\bmod (\varphi)($ resp., $\bmod (\Sigma))$, and logical consequence $\varphi \vDash \psi$ (resp., $\Sigma \vDash \psi$ ) are defined as usual. For two models $v$ and $w$, we denote by $v \leq w$ the usual componentwise ordering, i.e., $v_{i} \leq w_{i}$ for all $i=1,2, \ldots, n$, where $0 \leq 1 ; v<w$ means $v \neq w$ and $v \leq w$. Denote by $v \wedge w$ componentwise AND of models $v, w \in\{0,1\}^{n}$, and by $C l_{\wedge}(\mathcal{M})$ the closure of $\mathcal{M} \subseteq\{0,1\}^{n}$ under $\wedge$. Then, a theory $\Sigma$ is Horn representable if and only if $\bmod (\Sigma)=C l_{\wedge}(\bmod (\Sigma))$ (see $[2,9]$ ) for proofs).

Example 1. Consider $\mathcal{M}_{1}=\{(0101),(1001),(1000)\}$ and $\mathcal{M}_{2}=\{(0101),(1001)$, (1000), (0001), (0000)\}. Then, for $v=(0101), w=(1000)$, we have $w, v \in \mathcal{M}_{1}$, while $v \wedge w=(0000) \notin \mathcal{M}_{1}$; hence $\mathcal{M}_{1}$ is not the set of models of a Horn theory. On the other hand, $C l_{\wedge}\left(\mathcal{M}_{2}\right)=\mathcal{M}_{2}$, thus $\mathcal{M}_{2}=\bmod (\Sigma)$ for some Horn theory $\Sigma$. 
As discussed by Kautz et al. [7], a Horn theory $\Sigma$ is semantically represented by its characteristic models, where $v \in \bmod (\Sigma)$ is called characteristic (or extreme [2]), if $v \notin C l_{\wedge}(\bmod (\Sigma) \backslash\{v\})$. The set of all such models, the characteristic set of $\Sigma$, is denoted by $\operatorname{chr}(\Sigma)$. Note that $\operatorname{chr}(\Sigma)$ is unique. E.g., $(0101) \in \operatorname{chr}\left(\Sigma_{2}\right)$, while $(0000) \notin \operatorname{chr}\left(\Sigma_{2}\right)$; we have $\operatorname{chr}\left(\Sigma_{2}\right)=\mathcal{M}_{1}$. It is known [7] that the deductive query for a Horn theory $\Sigma$ from the characteristic set $\operatorname{chr}(\Sigma)$ can be done in linear time, i.e., $O(n|\operatorname{ch} r(\Sigma)|)$ time.

Interior and Exterior of Theories For a model $v \in\{0,1\}^{n}$ and an integer $\alpha>0$, its $\alpha$-neighborhood is defined by

$$
\mathcal{N}_{\alpha}(v)=\left\{w \in\{0,1\}^{n}\|w-v\| \leq \alpha\right\},
$$

where $\|v\|$ denotes $\sum_{i=1}^{n}\left|v_{i}\right|$. For a theory $\Sigma$ and an integer $\alpha>0$, the $\alpha$-interior and $\alpha$-exterior of $\Sigma$, denoted by $\sigma_{-\alpha}(\Sigma)$ and $\sigma_{\alpha}(\Sigma)$ respectively, are theories defined by

$$
\begin{aligned}
\bmod \left(\sigma_{-\alpha}(\Sigma)\right) & =\left\{v \in\{0,1\}^{n} \mid \mathcal{N}_{\alpha}(v) \subseteq \bmod (\Sigma)\right\} \\
\bmod \left(\sigma_{\alpha}(\Sigma)\right) & =\left\{v \in\{0,1\}^{n} \mid \mathcal{N}_{\alpha}(v) \cap \bmod (\Sigma) \neq \emptyset\right\} .
\end{aligned}
$$

By definition, $\sigma_{0}(\Sigma)=\sigma, \sigma_{\alpha}(\Sigma) \vDash \sigma_{\beta}(\Sigma)$ for integers $\alpha$ and $\beta$ with $\alpha<\beta$, and $\sigma_{\alpha}\left(\Sigma_{1}\right) \vDash \sigma_{\alpha}\left(\Sigma_{2}\right)$ holds for any integer $\alpha$, if two theories $\Sigma_{1}$ and $\Sigma_{2}$ satisfy $\Sigma_{1} \vDash \Sigma_{2}$.

Example 2. Let us consider a Horn theory $\Sigma=\left\{\bar{x}_{1} \vee x_{3}, \bar{x}_{2} \vee x_{3}, \bar{x}_{2} \vee x_{4}\right\}$ of 4 variables, where $\bmod (\Sigma)$ is given by

$$
\bmod (\Sigma)=\{(1111),(1011),(1010),(0111),(0011),(0010),(0001),(0000)\}
$$

(See Figure 2). Then we have $\sigma_{\alpha}(\Sigma)=\{\emptyset\}$ for $\alpha \leq-2,\left\{\bar{x}_{1}, \bar{x}_{2}, x_{3}, x_{4}\right\}$ for $\alpha=-1$, $\Sigma$ for $\alpha=0,\left\{\bar{x}_{1} \vee \bar{x}_{2} \vee x_{3} \vee x_{4}\right\}$ for $\alpha=1$, and $\emptyset$ for $\alpha \geq 2$. For example, $(0011)$ is the unique model of $\bmod \left(\sigma_{-1}(\Sigma)\right)$, since $\mathcal{N}_{1}(0011) \subseteq \bmod (\Sigma)$ and $\mathcal{N}_{1}(v) \nsubseteq \bmod (\Sigma)$ holds for all the other models $v$. For the 1-exterior, we can see that all models $v$ with $\left(\bar{x}_{1} \vee \bar{x}_{2} \vee x_{3} \vee x_{4}\right)(v)=1$ satisfy $\mathcal{N}_{1}(v) \cap \bmod (\Sigma) \neq \emptyset$, and no other such model exists. For example, (0101) is a model of $\sigma_{1}(\Sigma)$, since $(0111) \in \mathcal{N}_{1}(0101) \cap \bmod (\Sigma)$. On the other hand, (1100) is not a model of $\sigma_{1}(\Sigma)$, since $\mathcal{N}_{1}(1100) \cap \bmod (\Sigma)=\emptyset$. Notice that $\sigma_{-1}(\Sigma)$ is Horn, while $\sigma_{1}(\Sigma)$ is not.

Makino and Ibaraki [11] introduced the interiors and exteriors to analyze stability of Boolean functions, and studied their basic properties and complexity issues on them (see also [13]). For example, it is known [11] that, for a theory $\Sigma$ and nonnegative integers $\alpha$ and $\beta, \sigma_{-\alpha}\left(\sigma_{-\beta}(\Sigma)\right)=\sigma_{-\alpha-\beta}(\Sigma), \sigma_{\alpha}\left(\sigma_{\beta}(\Sigma)\right)=$ $\sigma_{\alpha+\beta}(\Sigma)$, and

$$
\sigma_{\alpha}\left(\sigma_{-\beta}(\Sigma)\right) \vDash \sigma_{\alpha-\beta}(\Sigma) \vDash \sigma_{-\beta}\left(\sigma_{\alpha}(\Sigma)\right) .
$$




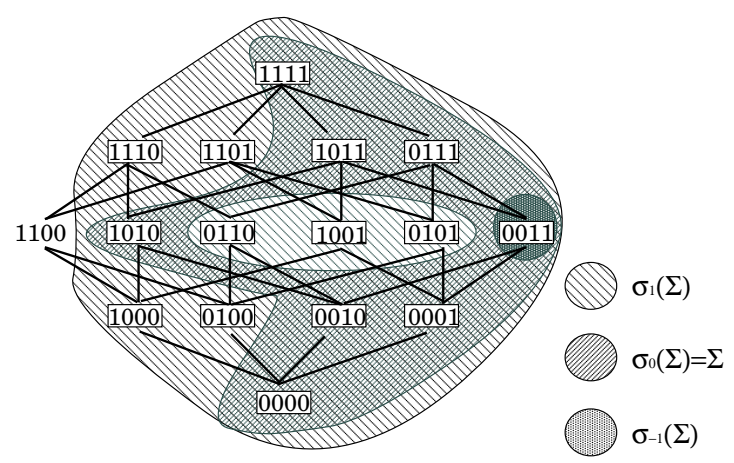

Fig. 2. A Horn theory and its interiors and exteriors

For an integer $\alpha>0$ and two theories $\Sigma_{1}$ and $\Sigma_{2}$, we have

$$
\begin{aligned}
\sigma_{-\alpha}\left(\Sigma_{1} \cup \Sigma_{2}\right) & =\sigma_{-\alpha}\left(\Sigma_{1}\right) \cup \sigma_{-\alpha}\left(\Sigma_{2}\right) \\
\sigma_{\alpha}\left(\Sigma_{1} \cup \Sigma_{2}\right) & \models \sigma_{\alpha}\left(\Sigma_{1}\right) \cup \sigma_{\alpha}\left(\Sigma_{2}\right),
\end{aligned}
$$

where $\sigma_{\alpha}\left(\Sigma_{1} \cup \Sigma_{2}\right) \neq \sigma_{\alpha}\left(\Sigma_{1}\right) \cup \sigma_{\alpha}\left(\Sigma_{2}\right)$ holds in general.

As demonstrated in Example 2, it is not difficult to see that the interiors of any Horn theory are Horn, which is, for example, proved by (4) and Lemma 1, while the exteriors might be not Horn.

\section{Deductive Inference from Horn Theories}

In this section, we investigate the deductive inference for the interiors and exteriors of a given Horn theory.

\subsection{Interiors}

Let us first consider the deduction for the $\alpha$-interiors of a Horn theory: Given a Horn theory $\Sigma$, a clause $c$, and a positive integer $\alpha$, decide if $\sigma_{-\alpha}(\Sigma) \vDash c$ holds. We show that the problem is solvable in linear time after showing a series of lemmas.

The following lemma is a basic property of the interiors.

Lemma 1. Let $c$ be a clause. Then for an integer $\alpha>0$, we have $\sigma_{-\alpha}(c)=$ $\bigvee_{\substack{S \subseteq c: \\|S|=\alpha+1}}\left(\bigwedge_{\ell \in S} \ell\right)=\bigwedge_{\substack{S \subseteq c: \\|S|=|c|-\alpha}}\left(\bigvee_{\ell \in S} \ell\right)$. 
This, together with (4), implies that for a $\operatorname{CNF} \varphi$ and an integer $\alpha>0$, we have

$$
\sigma_{-\alpha}(\varphi)=\bigwedge_{c \in \varphi}\left(\bigvee_{\substack{S \subseteq c: \\|S|=\alpha+1}}\left(\bigwedge_{\ell \in S} \ell\right)\right)=\bigwedge_{c \in \varphi}\left(\bigwedge_{\substack{S \subseteq c: \\|S||c|-\alpha}}\left(\bigvee_{\ell \in S} \ell\right)\right),
$$

where we regard $c$ as a set of literals.

Lemma 2. Let $\Sigma$ be a Horn theory, and let c be a clause. For an integer $\alpha>0$, if there exists a clause $d \in \Sigma$ such that $|N(d) \backslash N(c)| \leq \alpha-1$ or $(|N(d) \backslash N(c)|=\alpha$ and $P(d) \subseteq P(c)$ ), then we have $\sigma_{-\alpha}(\Sigma) \vDash c$.

Lemma 3. Let $\Sigma$ be a Horn theory, and let c be a clause. For an integer $\alpha>0$, if (i) $|N(d) \backslash N(c)| \geq \alpha$ holds for all $d \in \Sigma$ and (ii) $\emptyset \neq P(d) \subseteq N(c)$ holds for all $d \in \Sigma$ with $|N(d) \backslash N(c)|=\alpha$, then we have $\sigma_{-\alpha}(\Sigma) \mid \vDash c$.

By Lemmas 2 and 3, we can easily answer the deductive queries, if $\Sigma$ satisfies certain conditions mentioned in them. In the remaining case, we have the following lemma.

Lemma 4. For a Horn theory $\Sigma$ that satisfies none of the conditions in Lemmas 2 and 3 , let $d$ be a clause in $\Sigma$ such that $|N(d) \backslash N(c)|=\alpha$, and $P(d)=P(d) \backslash$ $(P(c) \cup N(c))=\{j\}$. Then $\sigma_{-\alpha}(\Sigma) \vDash c \vee x_{j}$ holds.

Proof. By Lemma 1, we have $\sigma_{-\alpha}(d) \vDash \bigvee_{i \in N(c) \cap N(d)} \bar{x}_{i} \vee x_{j} \vDash c \vee x_{j}$. This implies $\sigma_{-\alpha}(\Sigma) \vDash c \vee x_{j}$ by (4).

From this lemma, we have only to check $\sigma_{-\alpha}(\Sigma) \vDash c \vee \bar{x}_{j}$, instead of $\sigma_{-\alpha}(\Sigma) \vDash c$. Since $|c|<\left|c \vee \bar{x}_{j}\right| \leq n$, we can answer the deduction by checking the conditions in Lemmas 2 and 3 at most $n$ times.

We can see that a straightforward implementation of the algorithm requires $O(n(\|\Sigma\|+|c|))$ time, where $\|\Sigma\|$ denotes the length of $\Sigma$, i.e., $\|\Sigma\|=\sum_{d \in \Sigma}|d|$, though we can implement a linear time algorithm by adopting a proper data structure.

Theorem 1. Given a Horn theory $\Sigma$, a clause $c$ and an integer $\alpha>0$, a deductive query $\sigma_{-\alpha}(\Sigma) \vDash c$ can be answered in linear time, i.e., $O(\|\Sigma\|+|c|)$ time.

\subsection{Exteriors}

Let us next consider the deduction for the $\alpha$-exteriors of a Horn theory. In contrast to the interior case, we have the following negative result.

Theorem 2. Given a Horn theory $\Sigma$, a clause $c$ and a positive integer $\alpha$, it is co-NP-complete to decide whether a deductive query $\sigma_{\alpha}(\Sigma) \vDash c$ holds, even if $P(c)=\emptyset$. 


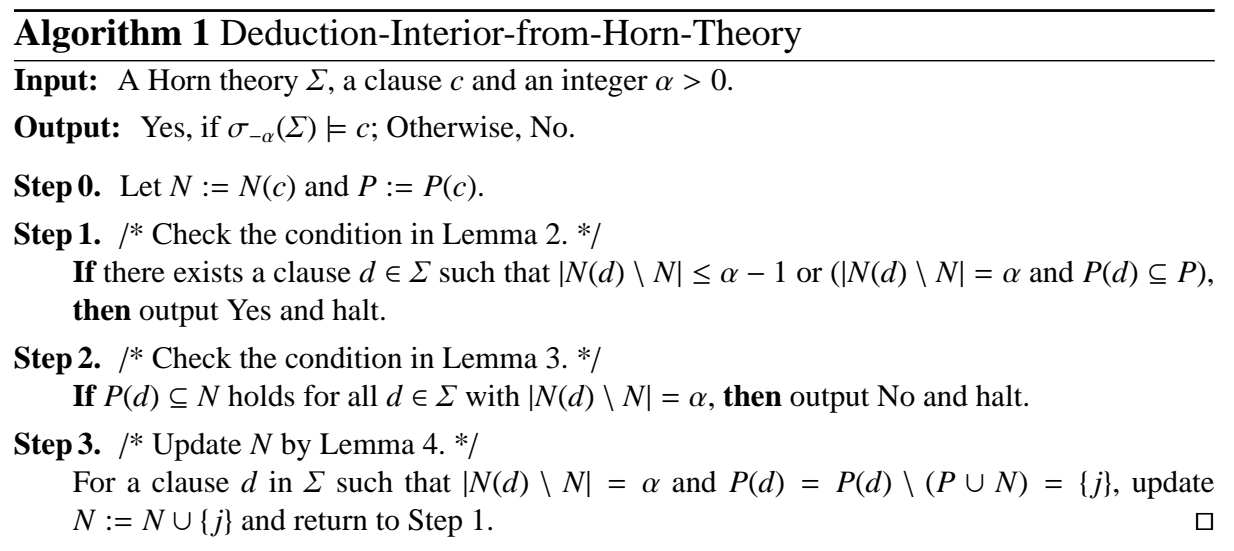

We remark that this result can also be derived from the ones in [11].

However, by using the next lemma, a deductive query can be answered in polynomial time, if $\alpha$ or $N(c)$ is small.

Lemma 5. Let $\Sigma_{1}$ and $\Sigma_{2}$ be theories. For an integer $\alpha>0$, Then $\sigma_{\alpha}\left(\Sigma_{1}\right) \vDash \Sigma_{2}$ if and only if $\Sigma_{1} \vDash \sigma_{-\alpha}\left(\Sigma_{2}\right)$.

From Lemma 5, the deductive query for the $\alpha$-interior of a theory $\Sigma$, i.e., $\sigma_{\alpha}(\Sigma) \vDash c$ for a given clause $c$ is equivalent to the condition that $\Sigma \vDash \sigma_{-\alpha}(c)$. Since we have $\sigma_{-\alpha}(c)=\bigwedge_{\substack{S \mid c: \text { : } \\|S|=|c|-\alpha}}\left(\bigvee_{\ell \in S} \ell\right)$ by Lemma 1 , the deductive query for the $\alpha$-interior can be done by checking $\left(\begin{array}{c}|c| \\ \alpha\end{array}\right)$ deductions for $\Sigma$. More precisely, we have the following lemma.

Lemma 6. Let $\Sigma$ be a Horn theory, let $c$ be a clause, and $\alpha>0$ be an integer. Then $\sigma_{\alpha}(\Sigma) \vDash c$ holds if and only if, for each subset $S$ of $N(c)$ such that $|S| \geq$ $|N(c)|-\alpha$, at least $(\alpha-|N(c)|+|S|+1) j$ 's in $P(c)$ satisfy $\Sigma \vDash \bigvee_{i \in S} \bar{x}_{i} \vee x_{j}$.

This lemma implies that the deductive query can be answered by checking the number of $j$ 's in $P(c)$ that satisfy $\Sigma \vDash \bigvee_{i \in S} \bar{x}_{i} \vee x_{j}$ for each $S$. Since we can check this condition in linear time and there are $\sum_{p=0}^{\alpha}\left(\begin{array}{c}|N(c)| \\ p\end{array}\right)$ such $S$ 's, we have the following result, which complements Theorem 2 that the problem is intractable, even if $P(c)=\emptyset$.

Theorem 3. Let $\Sigma$ be a Horn theory, let $c$ be a clause, and let $\alpha>0$ be an integer. Then a deductive query $\sigma_{\alpha}(\Sigma) \vDash c$ can be answered in $O\left(\sum_{p=0}^{\alpha}\left(\begin{array}{c}|N(c)| \\ p\end{array}\right) \|\right.$ $\Sigma \|+|P(c)|)$ time. In particular, it is polynomially solvable, if $\alpha=O(1)$ or $|N(c)|=O(\log \|\Sigma\|)$. 


\section{Deductive Inference from Characteristic Sets}

In this section, we consider the case when Horn knowledge bases are represented by characteristic sets. Different from formula-based representation, the deductions for interiors and exteriors are both intractable, unless $\mathrm{P}=\mathrm{NP}$.

\subsection{Interiors}

We first present an algorithm to solve the deduction problem for the interiors of Horn theories. The algorithm requires exponential time in general, but it is polynomial when $\alpha$ is small.

Let $\Sigma$ be a Horn theory given by its characteristic set $\operatorname{chr}(\Sigma)$, and let $c$ be a clause. Then for a nonnegative integer $\alpha$, we have

$$
\sigma_{-\alpha}(\Sigma) \vDash c \text { if and only if } \sigma_{-\alpha}(\Sigma) \wedge \bar{c} \equiv 0 .
$$

Let $v^{*}$ be a unique minimal model such that $c\left(v^{*}\right)=0$ (i.e., $\bar{c}\left(v^{*}\right)=1$ ). By the definition of interiors, $v^{*}$ is a model of $\sigma_{-\alpha}(\Sigma)$ if and only if all $v$ 's in $\mathcal{N}_{\alpha}\left(v^{*}\right)$ are models of $\Sigma$. Therefore, for each model $v$ in $\mathcal{N}_{\alpha}\left(v^{*}\right)$, we check if $v \in \bmod (\Sigma)$, which is equivalent to

$$
\bigwedge_{\substack{w \in \operatorname{chr}(\Omega) \\ w \geq v}} w=v
$$

If (7) holds for all models $v$ in $\mathcal{N}_{\alpha}\left(v^{*}\right)$, then we can immediately conclude by (6) that $\sigma_{-\alpha}(\Sigma) \not \neq c$. On the other hand, if there exists a model $v$ in $\mathcal{N}_{\alpha}\left(v^{*}\right)$ such that (7) does not hold, let $J=O N\left(\bigwedge_{\substack{w \in c h r(\Sigma) \\ w \geq v}} w\right) \backslash O N(v)$. By definition, we have $J \neq \emptyset$, and we can see that

$$
\sigma_{-\alpha}(\Sigma) \vDash \bigvee_{i \in O N(v)} \bar{x}_{i} \vee x_{j} \text { for all } j \in J .
$$

If $J \cap N(c) \neq \emptyset$, by Lemma 1 and (8), we have $\sigma_{-\alpha}(\Sigma) \vDash \bigvee_{i \in O N(v) \cap N(c)} \bar{x}_{i}$, since $|O N(v) \backslash N(c)| \leq \alpha-1$. This implies $\sigma_{-\alpha}(\Sigma) \vDash c$. On ther other hand, if $J \cap N(c)=\emptyset$, then by Lemma 1 and (8), we have $\sigma_{-\alpha}(\Sigma) \vDash \bigvee_{i \in N(c)} \bar{x}_{i} \vee x_{j}$ for all $j \in J$. Thus, if $J$ contains an index in $P(c)$, we can conclude that $\sigma_{-\alpha}(\Sigma) \vDash c$; Otherwise, we check the condition $\sigma_{-\alpha}(\Sigma) \vDash c \vee \bigvee_{j \in J} \bar{x}_{j}$, instead of $\sigma_{-\alpha}(\Sigma) \vDash c$.

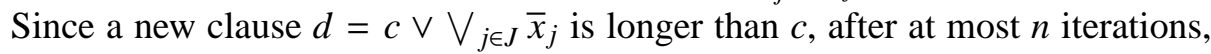
we can answer the deductive query. Formally, our algorithm can be described as Algorithm 2.

Theorem 4. Given the characteristic model $\operatorname{chr}(\Sigma)$ of a Horn theory $\Sigma$, a clause $c$ and a nonnegative integer $\alpha$, a deductive query $\sigma_{-\alpha}(\Sigma) \vDash c$ can be answered in $O\left(n^{\alpha+2}|\operatorname{chr}(\Sigma)|\right)$ time. In particular, it is polynomially solvable, if $\alpha=O(1)$. 


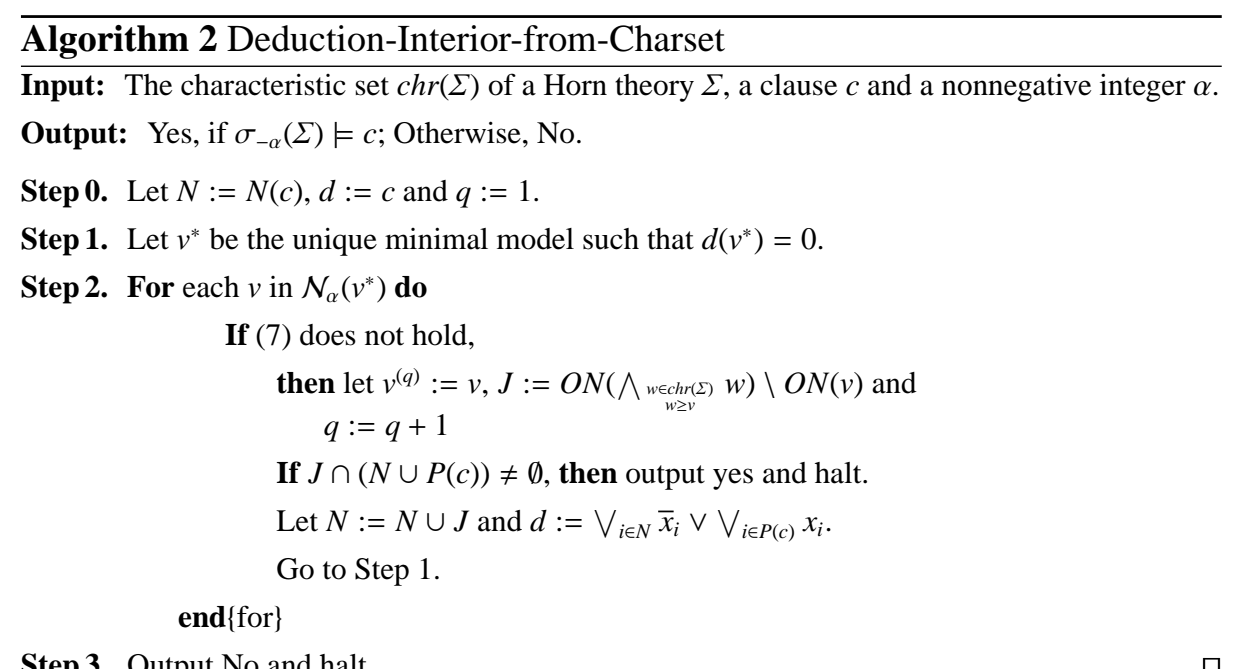

Step 3. Output No and halt.

However, in general, the problem is intractable, which contrasts to the formulamodel representation.

Theorem 5. Given the characteristic set $\operatorname{ch}(\Sigma)$ of a Horn theory $\Sigma$ and a positive integer $\alpha$, it is co-NP-complete to decide whether $\sigma_{-\alpha}(\Sigma)$ is consistent, i.e., $\bmod \left(\sigma_{-\alpha}(\Sigma)\right) \neq \emptyset$.

This result immediately implies the following corollary.

Corollary 1. Given the characteristic set $\operatorname{ch} r(\Sigma)$ of a Horn theory $\Sigma$, a clause $c$ and a positive integer $\alpha$, it is NP-complete to decide whether a deductive query $\sigma_{-\alpha}(\Sigma) \vDash c$ holds, even if $c=\emptyset$.

Note that, different from the other hardness results, the hardness is not sensitive to the size of $c$.

\subsection{Exteriors}

Let us consider the exteriors. Similarly to the formula-based representation, we have the following negative result.

Theorem 6. Given the characteristic set $\operatorname{ch}(\Sigma)$ of a Horn theory $\Sigma$, a clause $c$ and a positive integer $\alpha$, it is co-NP-complete to decide if a deductive query $\sigma_{\alpha}(\Sigma) \vDash c$ holds. 
By using Lemma 6, we can see that the problem can be solved in polynomial time, if $\alpha$ or $|N(c)|$ is small. Namely, for each subset $S$ of $N(c)$ such that $|S| \geq$ $|N(c)|-\alpha$, let $v^{S}$ denotes the model such that $O N\left(v^{S}\right)=S$. Then $w^{S}=\bigwedge_{\substack{w \in \operatorname{cr} r(\Sigma): \\ w \geq v^{S}}} w$ is the unique minimal model of $\Sigma$ such that $O N\left(w^{S}\right) \supseteq S$, and hence it follows from Lemma 6 that it is enough to check if $\left|O N\left(w^{s}\right) \cap P(c)\right| \geq \alpha-|N(c)|+|S|+1$. Clearly, this can be done in in $O\left(\sum_{p=0}^{\alpha}\left(\begin{array}{c}|N(c)| \\ p\end{array}\right) n|\operatorname{chr}(\Sigma)|\right)$ time.

Moreover, if $|P(c)|$ is small, then the problem also becomes tractable, which contrasts with Theorem 2 .

Lemma 7. Let $\Sigma$ be a Horn theory, let $c$ be a clause, and $\alpha$ be a nonnegative integer. Then $\sigma_{\alpha}(\Sigma) \vDash c$ holds if and only if each $S \subseteq P(c)$ such that $|S| \geq$ $|P(c)|-\alpha$ satisfies

$$
|O F F(w) \cap N(c)| \geq \alpha-|P(c)|+|S|+1
$$

for all models $w$ of $\Sigma$ such that $O F F(w) \cap P(c)=S$.

Note that (9) is monotone in the sense that, if a model $w$ satisfies (9), then all models $v$ with $v<w$ also satisfy it. Thus it is sufficient to check if (9) holds for all maximal models $w$ of $\Sigma$ such that $O F F(w) \cap P(c)=S$. Since such maximal models $w$ can be obtained from $w^{(i)}(i \in S)$ with $i \in O F F\left(w^{(i)}\right) \cap P(c) \subseteq S$ by their intersection $w=\bigwedge_{i \in S} w^{(i)}$, we can answer the deduction problem in $O\left(n \sum_{p=|P(c)|-\alpha}^{|P(c)|}\left(\begin{array}{c}|P(c)| \\ p\end{array}\right)|\operatorname{chr}(\Sigma)|^{p}\right)$ time.

Theorem 7. Given the characteristic set $\operatorname{chr}(\Sigma)$ of a Horn theory, a clause $c$, and an integer $\alpha \geq 0$, a deductive query $\sigma_{\alpha}(\Sigma) \vDash c$ can be answered in $O\left(n \min \left\{\sum_{p=0}^{\alpha}\left(\begin{array}{c}|N(c)| \\ p\end{array}\right)|\operatorname{chr} r(\Sigma)|, \sum_{p=|P(c)|-\alpha}^{|P(c)|}\left(\begin{array}{c}|P(c)| \\ p\end{array}\right)|\operatorname{chr}(\Sigma)|^{p}\right\}\right)$ time. In particular, it is polynomially solvable, if $\alpha=O(1),|P(c)|=O(1)$, or $|N(c)|=O(\log n$. $|\operatorname{chr}(\Sigma)|)$.

\section{Deductive Inference for Envelopes of the Exteriors of Horn Theories}

We have considered the deduction for the interiors and exteriors of Horn theories. As mentioned before, the interiors of Horn theories are also Horn, while this does not hold for the exteriors. This means that the exteriors of Horn theories might lose beneficial properties of Horn theories. One of the ways to overcome such a hurdle is Horn Approximation, that is, approximating a theory by a Horn theory [16]. There are several methods for approximation, but one of the most natural ones is to approximate a theory by its Horn envelope. For a theory $\Sigma$, its Horn envelope is the Horn theory $\Sigma_{e}$ such that $\bmod \left(\Sigma_{e}\right)=C l_{\wedge}(\bmod (\Sigma))$. 
Since Horn theories are closed under intersection, Horn envelope is the least Horn upper bound for $\Sigma$, i.e., $\operatorname{chr}\left(\Sigma_{e}\right) \supseteq \operatorname{chr}(\Sigma)$ and there exists no Horn theory $\Sigma^{*}$ such that $\operatorname{chr}\left(\Sigma_{e}\right) \supsetneq \operatorname{chr}\left(\Sigma^{*}\right) \supseteq \operatorname{chr}(\Sigma)$. In this section, we consider the deduction for Horn envelopes of interiors of Horn theories; $\sigma_{\alpha}(\Sigma)_{e} \vDash c$.

\subsection{Model-Based Representations}

Let us first consider the case in which knowledge bases are represented by characteristic sets.

Proposition 1. Let $\Sigma$ be a Horn theory, and let $\alpha$ be a nonnegative integer. Then we have

$$
\bmod \left(\sigma_{\alpha}(\Sigma)_{e}\right)=C l_{\wedge}\left(\bigcup_{v \in \operatorname{chr}(\Sigma)} \mathcal{N}_{\alpha}(v)\right)
$$

For a clause $c$, let $v^{*}$ be the unique minimal model such that $c\left(v^{*}\right)=0$. We recall that, for a Horn theory $\Phi$,

$$
\Phi \vDash c \text { if and only if } c\left(\bigwedge_{\substack{v \in c h r(\Phi) \\ v \geq v^{*}}} v\right)=1 .
$$

Therefore, Proposition 1 immediately implies an algorithm for the deduction for $\sigma_{\alpha}(\Sigma)_{e}$ from $\operatorname{chr}(\Sigma)$, since we have $\operatorname{chr}\left(\sigma_{\alpha}(\Sigma)_{e}\right) \subseteq \bigcup_{v \in \operatorname{chr}(\Sigma)} \mathcal{N}_{\alpha}(v)$. However, for a general $\alpha, \bigcup_{v \in \operatorname{chr}(\Sigma)} \mathcal{N}_{\alpha}(v)$ is exponentially larger than $\operatorname{chr}(\Sigma)$, and hence this direct method is not efficient. The following lemma helps developing a polynomial time algorithm.

Lemma 8. Let $\Sigma$ be a Horn theory, let c be a clause, and let $\alpha$ be a nonnegative integer. Then $\sigma_{\alpha}(\Sigma)_{e} \vDash c$ holds if and only if the following two conditions are satisfied.

(i) $|O F F(v) \cap N(c)| \geq \alpha$ holds for all $v \in \operatorname{chr}(\Sigma)$.

(ii) If $S=\{v \in \operatorname{chr}(\Sigma)|| O F F(v) \cap N(c) \mid=\alpha\} \neq \emptyset, P(c)$ is not covered with $O F F(v)$ for models $v$ in $S$, i.e., $P(c) \nsubseteq \bigcup_{\substack{v \in \operatorname{ch}(\Omega) \\|O F F(v) \cap N(c)|=\alpha}} O F F(v)$.

The lemma immediately implies the following theorem.

Theorem 8. Given the characteristic set $\operatorname{chr}(\Sigma)$ of a Horn theory $\Sigma$, a clause c, and an integer $\alpha \geq 0$, a deductive query $\sigma_{\alpha}(\Sigma)_{e} \vDash c$ can be answered in linear time.

We remark that this contrasts with Corollary 1 . Namely, if we are given the characteristic set $\operatorname{chr}(\Sigma)$ of a Horn theory $\Sigma, \sigma_{\alpha}(\Sigma)_{e} \vDash c$ is polynomially solvable, while it is co-NP-complete to decide if $\sigma_{\alpha}(\Sigma) \vDash c$. 


\subsection{Formula-Based Representation}

Recall that a negative theory (i.e., a theory consisting of clauses with no positive literal) is Horn and the exteriors of negative theory are also negative, and hence Horn. This means that, for a negative theory $\Sigma$, we have $\sigma_{\alpha}(\Sigma)_{e}=\sigma_{\alpha}(\Sigma)$. Therefore, we can again make use of the reduction in the proof of Theorem 2, since the reduction uses negative theories.

Theorem 9. Given a Horn theory $\Sigma$, a clause $c$, and an integer $\alpha \geq 0$, it is co-NP-complete to decide whether $\sigma_{\alpha}(\Sigma)_{e} \vDash c$ holds, even if $P(c)=\emptyset$.

However, if $\alpha$ or $N(c)$ is small, the problem becomes tractable by Algorithm 3.

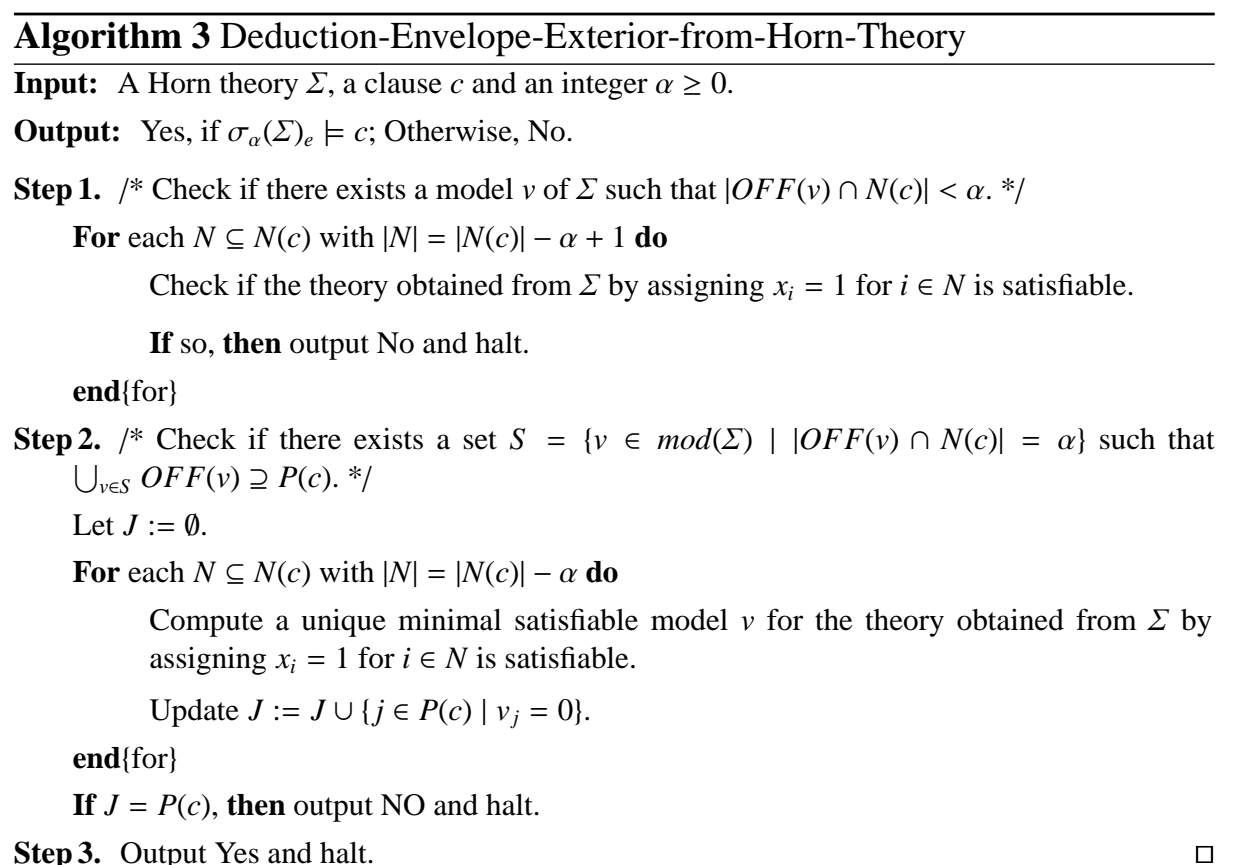

The algorithm is based on a necessary and sufficient condition for $\sigma_{\alpha}(\Sigma)_{e} \vDash$ $c$, which is obtained from Lemma 8 by replacing all $\operatorname{chr}(\Sigma)$ 's with $\bmod (\Sigma)$ 's. It is not difficult to see that such a condition holds from the proof of Lemma 8.

Theorem 10. Given a Horn theory $\Sigma$, a clause c, and an integer $\alpha \geq 0$, a deductive query $\sigma_{\alpha}(\Sigma)_{e} \vDash c$ can be answered in $O\left(\left(\left(\begin{array}{c}|N(c)| \\ \alpha-1\end{array}\right)+\left(\begin{array}{c}|N(c)| \\ \alpha\end{array}\right)\right)\|\Sigma\|+|P(c)|\right)$ 
time. In particular, it is polynomially solvable, if $\alpha=O(1)$ or $|N(c)|=O(\log \|$ $\Sigma \|)$.

\section{References}

1. R. J. Brachman and H. J. Levesque. Knowledge Representation and Reasoning. Elsevier, 2004.

2. R. Dechter and J. Pearl. Structure identification in relational data. Artificial Intelligence, 58 (1992) 237-270.

3. W. Dowling and J. H. Galliear. Linear-time algorithms for testing the satisfiability of propositional Horn theories. Journal of Logic Programming 3 (1983) 267-284.

4. T. Eiter, T. Ibaraki, and K. Makino. Computing intersections of Horn theories for reasoning with models. Artificial Intelligence, 110 (1999) 57-101.

5. T. Eiter and K. Makino. On computing all abductive explanations. In Proc. AAAI-2002 (2002) 62-67.

6. D. Kavvadias, C. Papadimitriou, and M. Sideri. On Horn Envelopes and Hypergraph Transversals. In Proc. ISAAC-93, LNCS 762, (1993) 399-405.

7. H. Kautz, M. Kearns, and B. Selman. Reasoning with characteristic models. In Proc. AAAI93 (1993) 34-39.

8. H. Kautz, M. Kearns, and B. Selman. Horn approximations of empirical data. Artificial Intelligence, 74 (1995) 129-245.

9. R. Khardon and D. Roth. Reasoning with models. Artificial Intelligence, 87 (1996) 187-213.

10. R. Khardon and D. Roth. Defaults and relevance in model-based reasoning. Artificial Intelligence, 97 (1997) 169-193.

11. K. Makino and T. Ibaraki, Interior and exterior functions of Boolean functions, Discrete Applied Mathematics, 69 (1996) 209-231.

12. K. Makino and H. Ono, Deductive Inference for the Interiors and Exteriors of Horn Theories, Mathematical Engineering Technical Report, METR 2007-06, The University of Tokyo, February 2007.

13. K. Makino, H. Ono and T. Ibaraki, Interior and exterior functions of positive Boolean functions, Discrete Applied Mathematics, 130 (2003) 417-436.

14. J. Makowsky. Why Horn formulas matter for computer science: Initial structures and generic examples. Journal of Computer and System Sciences, 34 (1987) 266-292.

15. J. McKinsey. The decision problem for some classes of sentences without quantifiers. Journal of Symbolic Logic, 8 (1943) 61-76.

16. B. Selman and H. Kautz, Knowledge compilation using Horn approximations, In Proc. AAAI-91, (1991) 904-909. 